\title{
Aufbau hochfunktionaler Intralogistik-Knoten mittels kleinskaliger Module als Cognitive Conveyor
}

\author{
Development of Highly-functional Nodes for Warehousing Applications by Using \\ Small-scaled Modules as Cognitive Conveyor
}

\author{
Kai Ventz* \\ M. Bechir Hachicha ** \\ Mišel Radosavac ** \\ Tobias Krühn ** \\ Ludger Overmeyer ** \\ * Technik \& Innovation, Transnorm System GmbH, Harsum \\ ** Institut für Transport- und Automatisierungstechnik, Leibniz Universität Hannover
}

$\mathbf{K}$ leinskalige, multifunktionale Module haben ein hohes Potential bei der wirtschaftlichen und flexiblen Gestaltung intralogistischer Systeme mit hoher Funktionalität. Durch dezentrale Steuerung und eigener Intelligenz der Module ist das System frei skalierbar und der Installationsaufwand wird minimiert. Mittels eines neuartigen Konzeptes der Datenkommunikation für Stetigförderer erfolgt der Informationsaustausch drahtlos mit Hilfe optoelektrischer Elemente. Die Kleinskaligkeit der Transportmodule gegenüber der Transporteinheit im Zusammenhang mit dem Steuerungskonzept erlaubt eine selektive Beschaltung der Module nach Bedarf und damit eine optimierte Energieausnutzung im Betrieb. Prototypen auf Basis von Schwenkrollen mit integrierter Antriebstechnik und Steuerung lassen das Potential des Prinzips erkennen. Das neu entwickelte Konzept der Schrägscheibe hilft bei der anspruchsvollen Integration der Antriebstechnik in das Modul durch das Prinzip der koaxialen Aktoren. Durch omnidirektionalen Funktionsumfang der Module entsteht im Zusammenschluss zu einer Modulmatrix ein hochflexibel einsetzbares Intralogistik-Modul. Die Vernetzung dieser hochfunktionalen Knoten durch einfache Fördertechnik bietet die Möglichkeit einfacher Planung flexibler Logistiksysteme.

[Schlüsselwörter: Intralogistik, kleinskalige Transportmodule, Transporttechnik, dezentrale Steuerung, Materialflusstechnik]

S mall-scaled modules offer a considerable potential for the economic and flexible design of warehousing systems with high functionality. By using distributed control systems with embedded intelligence in the modules, the whole system is freely scalable and the installation effort is reduced at the same time. The information exchange between continuous ground conveyors is performed by a novel concept for data communication by the use of opto-electrical elements. The scalability of the conveyor modules compared to the transport unit in combination with the control concept allows selective activation of required modules and therewith optimized energy efficiency. Prototypes based on swiveling rollers show the potential of the principle. The newly developed concept - using a tilting disc - supports the ambitious integration of drive systems in the module through the principle of coaxial actuators. The modules with omnidirectional functionality can be combined to a module matrix from which a highly flexible module is derived. Cross-linking these highly functional nodes by simple material handling systems offer the possibility for simple planning of flexible warehousing systems.

[Keywords: warehousing systems, small scaled conveyor modules, Cognitive Conveyor, material handling technology]

\section{EINLEITUNG}

Die innerbetriebliche Materialflusstechnik als Teil der Intralogistik-Branche unterliegt dem Einfluss nachhaltiger Trends, wie kürzeren Produktlebenszyklen oder stärkerer Individualisierung. Daraus resultiert als Anforderung an Materialflusssysteme in sämtlichen Produktionsund Logistikprozessen ein höheres Maß an Wandelbarkeit [De09]. Beobachtungen aus Sicht der Hersteller für intralogistische Systeme zeigen ein stetig steigendes Spektrum an Fördergut von kleinsten unverpackten Teilen bis zu großen Kartons und Behältern, die möglicherweise ein gemeinsames Materialflusssystem nutzen und deren Anforderungen an das System durchaus gegenläufig sind. Die Funktionalitäten werden bei der Planung von Intralogistik-Anlagen heute ganz speziell auf den Anwendungsfall zugeschnitten. Zudem sind die heute üblichen Materialflusssysteme zentral gesteuert. Mechanisch besitzen sie eine definierte Ausprägung, die weitgehend festliegt und somit sind auch die elementaren Funktionalitäten im System lokal gebunden, wie z. B. das Ausschleusen, Verteilen und Sortieren. Solche Funktionalitäten werden auf das 
größte im System zu bewegende Fördergut ausgelegt und bedeuten für kleineres Fördergut Leistungsverlust im System. Veränderungen der Topologie fördertechnischer Funktionen sind nur mit großem Aufwand möglich. Besonders Neuverkabelung und Neuprogrammierung der zentralen Steuerung sind ausgesprochen zeit- und kostenintensiv.

Ein modernes intralogistisches Konzept soll mit geringem Planungsaufwand flexibel, rekonfigurierbar und skalierbar sein, um Logistikprozesse nachhaltigen Veränderungen anpassen zu können. Es gibt bereits Ansätze gleichartige intralogistische Module mit komplett dezentraler Steuerung auszustatten [FM09]. Das Prinzip beruht auf Rollenfördertechnik mit integriertem Hubriemenausschleuser. Der Ansatz der kleinskaligen Module bietet eine höhere Flexibilität bei der Planung und im Zusammenhang mit einem geeigneten Steuerungskonzept eine höhere Funktionalität.

\section{GRUNDPRINZIP KLEINSKALIGER, MULTIFUNKTIONALER STETIGFÖRDERER}

Der Ansatz gleichartiger, kleinskaliger Förderkomponenten mit eingeprägter Intelligenz, wie in [Ov07] vorgestellt, stellt ein erhebliches Optimierungspotential dar. Abbildung 1 zeigt einen konventionellen Rollenförderer mit Ausschleuser und Anschlussförderer. Die Ausschleusfunktion ist bei einer derartigen, heute üblichen, Konfiguration lokal im System gebunden.
Das intralogistische Konzept der kleinskaligen Module ist dagegen flexibel auf Veränderungen anpassbar. Gleichzeitig bleibt der Investitionsaufwand vergleichsweise gering. Die einzelnen Module lösen im Zusammenschluss individuelle, komplexe Transportaufgaben und versprechen durch ihre Gleichartigkeit, bei vergleichsweise kleinen Abmessungen, produktionstechnische und damit Kostenvorteile. Je nach Größe der Module kann eine Transporteinheit ein oder mehrere Module sowohl in Längs- und Querrichtung belegen (vgl. Abbildung 1, Modulmatrix).

In Zusammenhang mit einem geeigneten Steuerungskonzept führt der Zusammenschluss der einzelnen Module zu einem Zuwachs an Funktionalität gegenüber einem Einzelmodul (Funktionsemergenz).Voraussetzung für die hohe Funktionalität der Matrix ist die omnidirektionale Funktionalität des einzelnen Moduls, d. h. das Modul kann fördertechnische Aufgaben in jeder beliebigen Richtung in der Ebene übernehmen (siehe Abbildung 2).

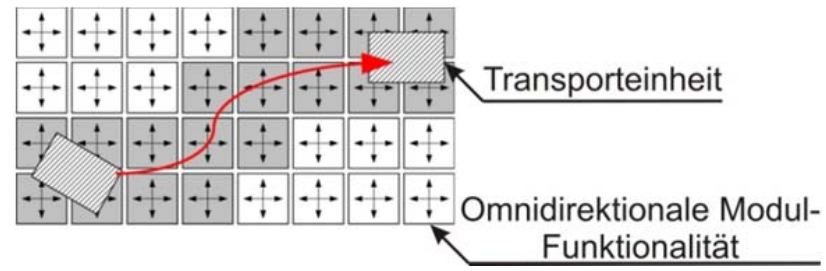

Abbildung 2. Modulmatrix aus omnidirektionalen Modulen

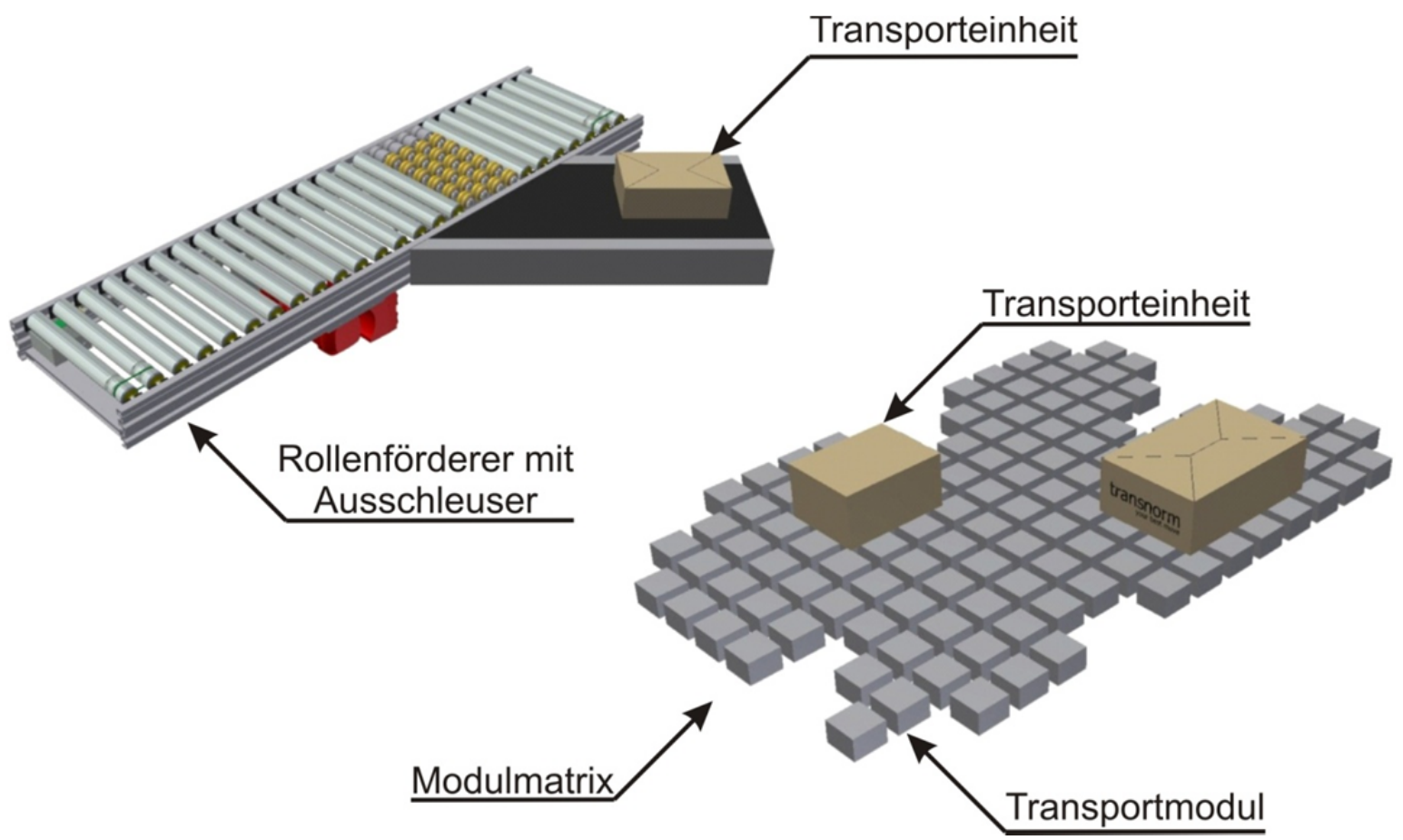

Abbildung 1. Konventionelle Ausschleustechnik (links) und kleinskalige Transportmodule (rechts) 
Ein wesentlicher Aspekt ist die Kleinskaligkeit des Moduls gegenüber der Transporteinheit:

- $\quad$ Eine feinere geometrische Auflösung erlaubt eine größere Freiheit bei der Abbildung unterschiedlicher Topologien bei der Planung und Gestaltung eines Systems.

- Durch die omnidirektionale Funktionalität und individuelle Operation der Einzelmodule werden neben Sortierfunktionen bei Belegung mehrerer Module zusätzliche Funktionen wie Drehen, Orientieren, Ausrichten, Puffern oder Sequenzieren möglich (siehe Abbildung 3).

- Im Vergleich zu größeren Modulen ist eine höhere Durchsatz- und Sortierleistung möglich, da die Abstände der Transporteinheiten im Betrieb minimiert werden können.

- Beim Zusammenschluss der Module zu einer Matrix sind die Funktionalitäten wie Ausschleusen oder Sortieren an jedem Punkt innerhalb der Matrix abrufbar und nicht wie in konventionellen Systemen lokal gebunden.

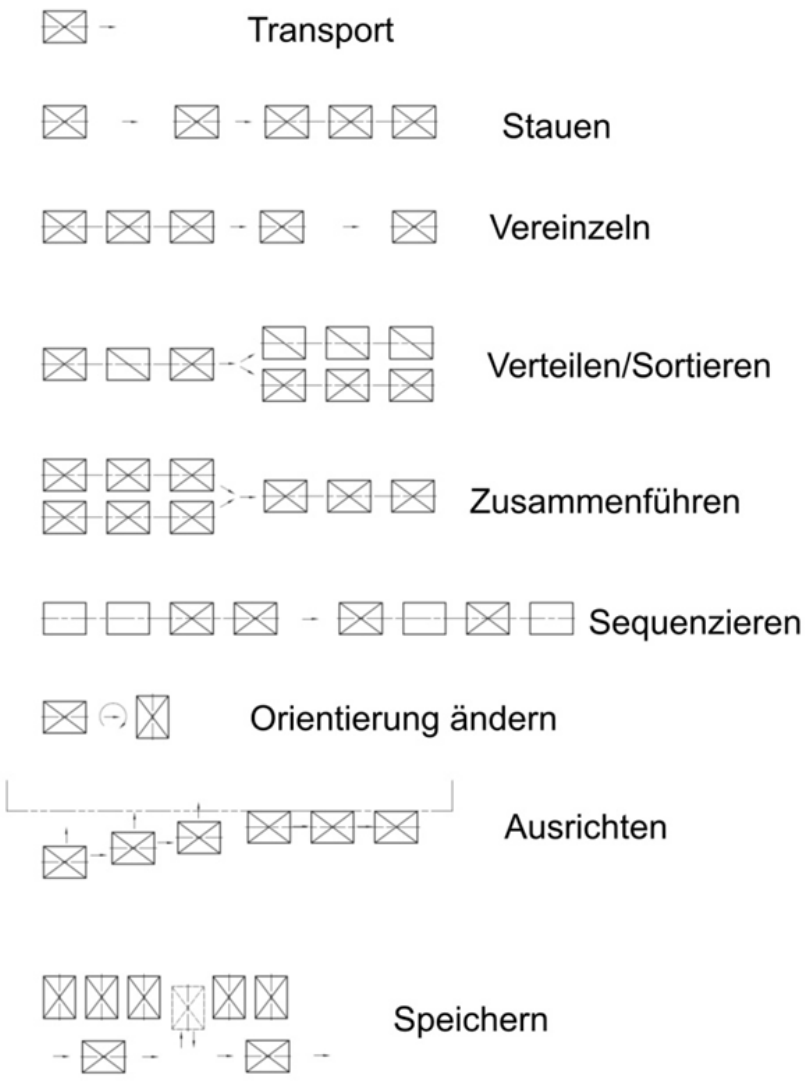

Abbildung 3. Intralogistische Funktionen einer Modulmatrix (schematisch)

\section{NEUE TECHNISCHE LÖSUNGEN ZUR GESTALTUNG KLEINSKALIGER MODULE}

Eine technische Lösung zur Realisierung kleinskaliger Stetigförderer mit omnidirektionaler Funktionalität bietet die Schwenkrollentechnik mit integrierter Antriebstechnik und Steuerung [OKV10]. Abbildung 4 zeigt den Aufbau des Schwenkrollenmoduls mit Rollenantrieb zum Fördern der Transporteinheit und Schwenkantrieb zur Richtungsänderung. Das Modul hat einem Rollendurchmesser von $50 \mathrm{~mm}$ und einer Grundfläche von 60 x 60 $\mathrm{mm}$.

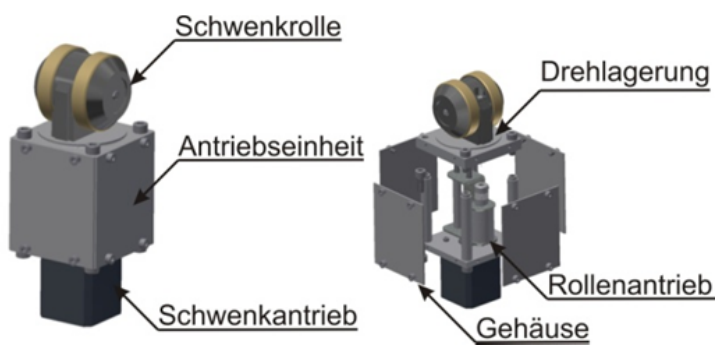

Abbildung 4. Modell des Schwenkrollenmoduls

Die auf der Schwenkrollentechnik basierenden Prototypen in Abbildung 5 zeigen in einem Demonstrator das Potential des Prinzips der Module. Die Integration der Antriebstechnik ist dabei aufgrund des begrenzten Bauvolumens und der benötigten Dynamik sehr anspruchsvoll.

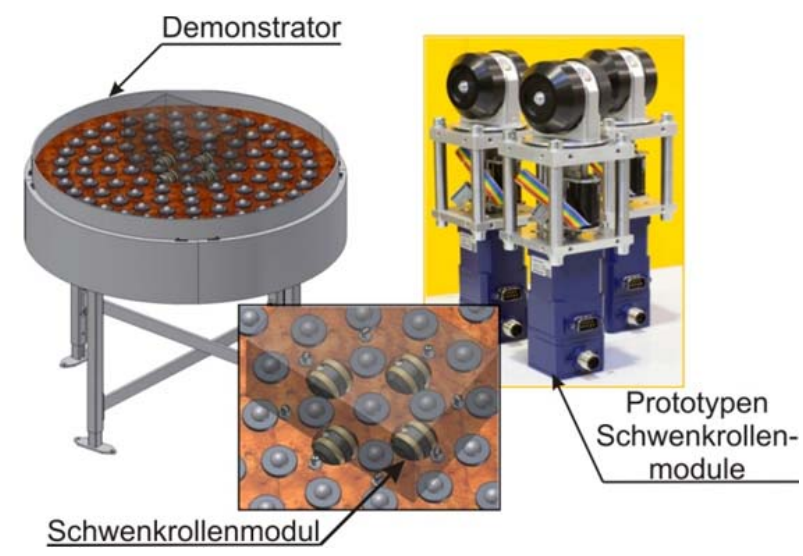

Abbildung 5. Modulare Stetigförderer-Prototypen auf Basis von Schwenkrollentechnik in einem Demonstrator

Bei einem weiteren Prototyp wurde das Prinzip der Schwenkscheibe umgesetzt (siehe Abbildung 6 und 7). Eine zur Förderebene schrägstehende, rotierende Scheibe sorgt für den Vortrieb der Transporteinheit, die auf mehreren Modulen aufliegt. Eine Verdrehung der rotierenden Scheibe mittels Schwenkantrieb verdreht die Scheibe um die Senkrechte und sorgt für eine Richtungsänderung beim Transport. Abbildung 6 und 7 zeigen den prinzipiellen Aufbau dieses Antriebskonzepts. 


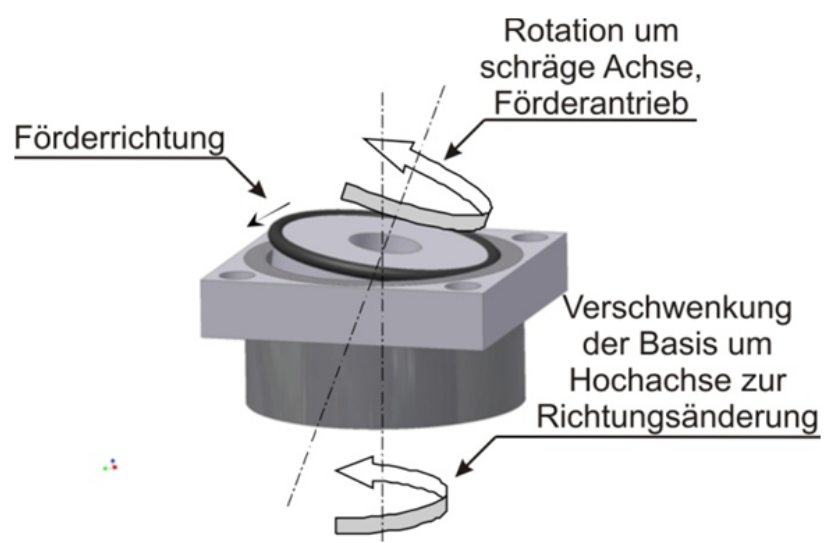

Abbildung 6. Funktionsprinzip der Schwenkscheibe

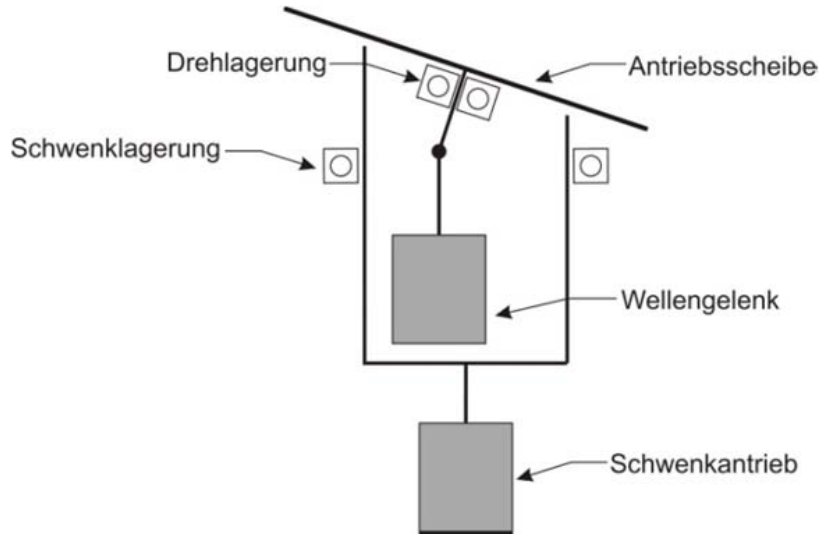

Abbildung 7. Anordnung der Antriebstechnik im Schwenkscheibenmodul

Das Konzept der Schwenkscheibe erlaubt eine koaxiale Anordnung der Antriebe und bietet bei der Forderung nach weniger Bauvolumen und Kompaktheit weiteres Potential.

Im Transportmodul wird zwischen der Steuerungseinheit und der mechanischen Einheit unterschieden. Als mechanische Einheit wird der Bereich des Transportmoduls bezeichnet, der die ganzen Antriebs- und Schwenkkomponenten enthält. Die Steuerungseinheit enthält hingegen alle Steuerungskomponenten und wird am unteren Teil des Transportmoduls angeordnet (siehe Abbildung 8) und im Gehäuse des Moduls aufgenommen. Jedes Modul ist mit einem Lichttaster zur Erfassung der Transporteinheiten ausgestattet. Der Zusammenbau des Moduls mit Gehäuse und Steuerungseinheit ist in Abbildung 9 zu sehen.

Um die Adaptivität und Flexibilität des Transportmoduls zu unterstützen, soll es ohne Aufwand anschließbar und austauschbar sein. Ein Haltemodul ermöglicht den einfachen Austausch und die Energieversorgung. Dieses Haltemodul ist mit einer Schraubenverbindung an der Transportmodulumgebung befestigt und stellt eine formschlüssige Verbindung mit dem Transportmodul her. Eine Spannverbindung mit einem Schiene-Wagen-System er- möglicht den mechanischen Zusammenschluss der benachbarten Module.

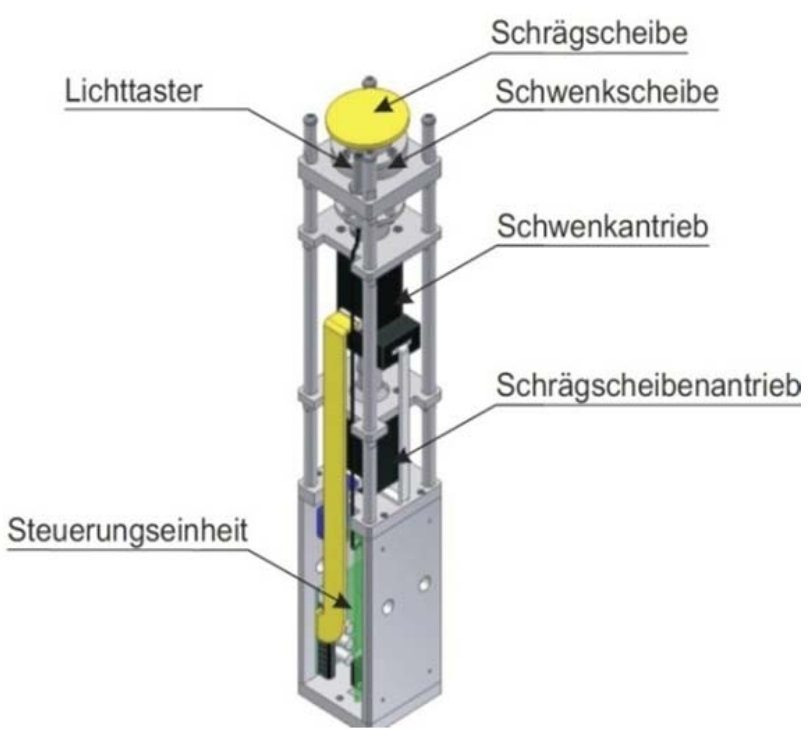

Abbildung 8. Prototyp Schwenkscheibenmodul

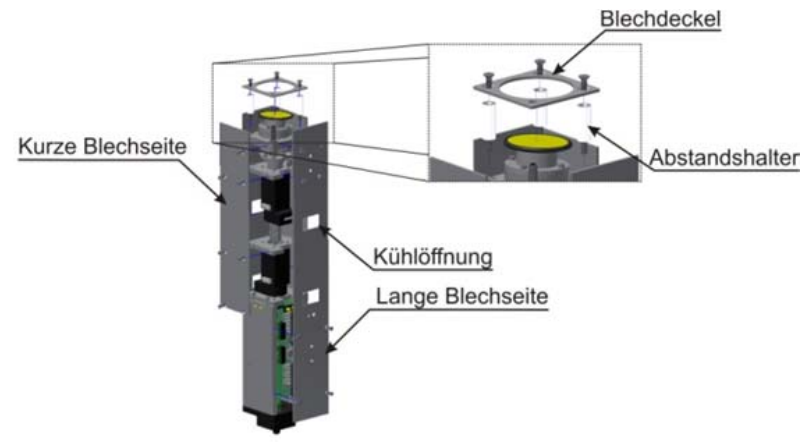

Abbildung 9. Zusammenbau Schrägscheibenmodul mit Gehäuse

Zur Energieversorgung werden ein Stecker am Haltemodul und eine Buchse am unteren Teil des Transportmoduls eingebaut. Eine Leitung verbindet die Buchse mit der Steuerungsplatine, um diese mit Spannung zu versorgen. Die Steuerungsplatine wird ebenfalls mit dem Transportmodul als Masse verbunden. Das untere Befestigungsteil am Transportmodul wird im Gegensatz zu anderen Teilen des Moduls aus Kunststoff gefertigt. Damit ist eine Abtrennung der Versorgungsspannung vom Massepotential realisiert. Abbildung 10 zeigt den prinzipiellen Aufbau der Energieversorgung für das Transportmodul. 


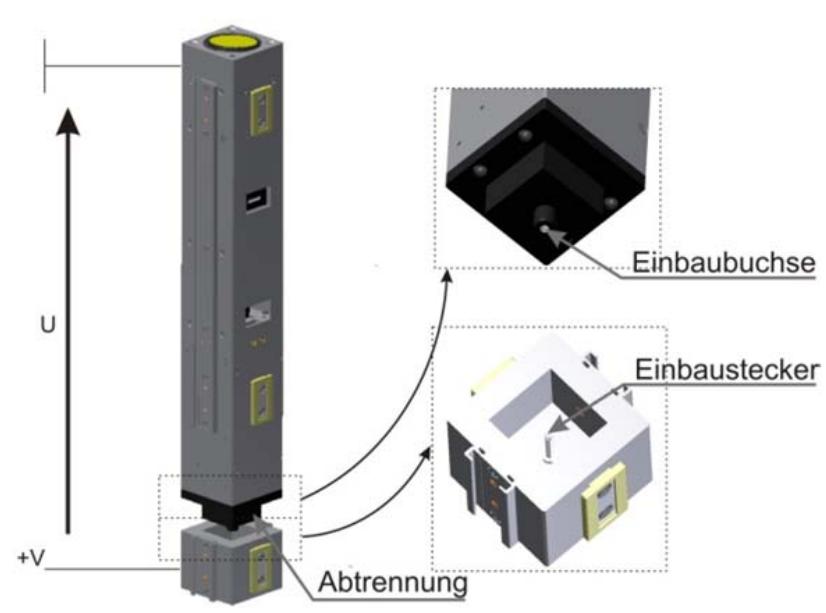

Abbildung 10. Energieversorgung der Module durch Steckkontakte

Die elektrische Energie wird durch das Haltemodul an die anderen Haltemodule weitergeleitet. Die Leitung der elektrischen Energie erfolgt mit dem Kontakt zwischen der Schiene und den Befestigungsschrauben der Wagen. Um diese Verbindung zu sichern und einen möglichen Luftspalt zu verhindern, wird an der Schiene eine zusätzliche Kontaktfeder auf der Höhe der Schrauben eingebaut. Die Kontaktfeder an der Schiene drückt auf die Befestigungsschraube der Wagen und stellt damit eine sichere Verbindung zwischen den beiden Elementen her. Auf gleiche Weise werden die Transportmodule miteinander verbunden, um das Massenpotential des Systems zu bilden. Abbildung 11 stellt diese Verbindung dar. Es genügt, wenn ein Transportmodul an die Betriebsspannung angeschlossen wird. Damit wird die elektrische Energie an alle Transportmodule weitergeleitet (siehe Abbildung 11). Abbildung 12 zeigt ein Beispiel des Zusammenschlusses der Transportmodule zu einer Funktionsmatrix.

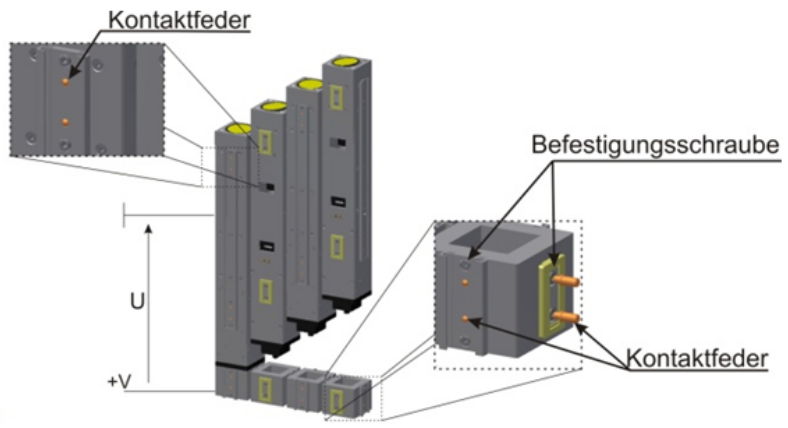

Abbildung 11. Weiterleitung der Energie durch integrierte Kontakte

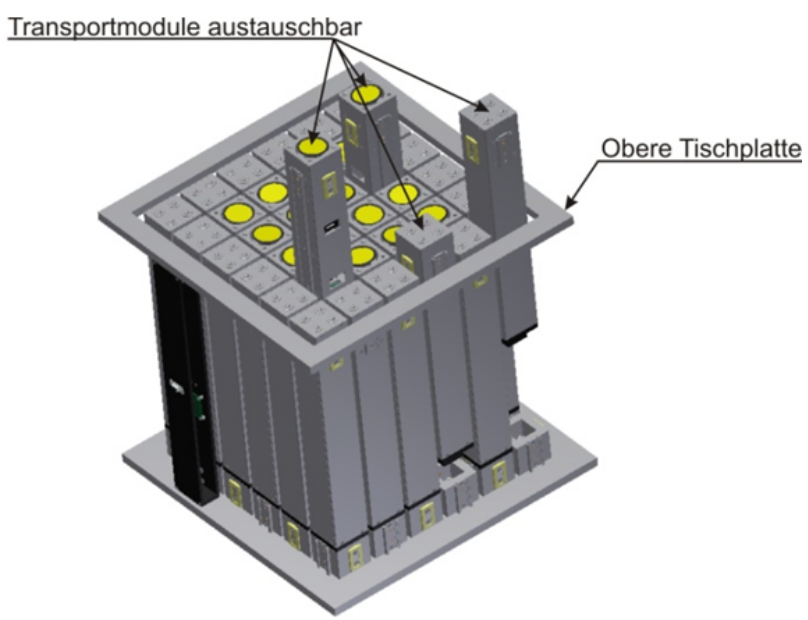

Abbildung 12. Zusammenschluss zur Funktionsmatrix

\section{STEUERUNGS- UND KOMMUNIKATIONSKONZEPT}

Das entwickelte, modular aufgebaute Transportmodul ist zur Erreichung einer hohen Flexibilität und Adaptivität mit einer dezentralen Intelligenz in Form einer eigenen Steuerung und Sensorik ausgestattet. Die Steuerungsplatine des Transportmoduls verbindet die unterschiedlichen Komponenten der steuerungstechnischen Ebene mit einem Mikrocontroller und der Energieversorgung. Abbildung 13 stellt den prinzipiellen Aufbau der Steuerungsplatine dar.

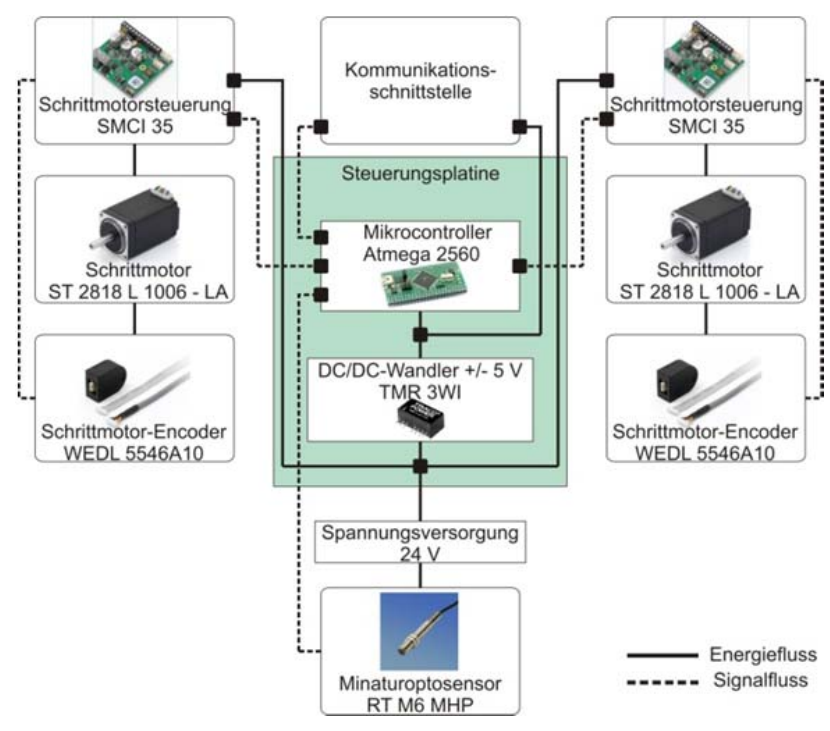

Abbildung 13. Struktur der Steuerungsplatine

Der Förderantrieb sowie der Schwenkantrieb werden über Steuerungscontroller mit der Bezeichnung SMCI 35 der Firma Nanotec Electronics gesteuert. Hierbei handelt es sich jeweils um eine Leistungsstufe, welche Drehzahl und Drehrichtung der Antriebe steuert. Außerdem ist an jedem Antrieb der Encoder des Typs WEDL 5546 - A10 angeschlossen, wodurch die exakte Positionierung der Antriebe ermöglicht wird. Die beiden Steuerungscontrol- 
ler sind mit der Steuerungsplatine, die mit dem Mikrocontroller des Typs Atmega 2560 ausgestattet ist, verbunden. Weiterhin ist ein Miniaturoptosensor mit der Steuerungsplatine verbunden und erkennt die Ankunft einer Transporteinheit auf dem Transportmodul.

Um Informationen sowie Daten über den Materialflussablauf zu empfangen bzw. zu senden und diese dezentral zu verarbeiten, soll das Transportmodul fähig sein mit benachbarten Modulen zu kommunizieren und das unmittelbar nach dem Einbau. Hierfür wird eine optische Kommunikationsschnittstelle verwendet, wobei eine Leucht-Photodioden-Paarung das Verbindungskabel zu dem benachbarten Transportmodul ersetzt und eine bidirektionale drahtlose Datenübertragung ermöglicht. Das Ergebnis ist ein geringer Verkabelungsaufwand und das Transportmodul ist nach dem Anschließen bereit zur Inbetriebnahme.

Die Schaltung für die Kommunikationsschnittstelle basiert auf einem Transimpedanzverstärker für die Photodiode und erreicht eine Bitrate von $1 \mathrm{MBit} / \mathrm{s}$. Um die Kommunikation eines Transportmoduls mit seinen vier benachbarten Modulen zu gewährleisten, darf der Abstand zwischen den Leuchtdioden und den Photodioden von unterschiedlichen Transportmodulen maximal so groß sein, dass noch ein visueller Kontakt der Bauelemente vorhanden ist. Daher werden jeweils eine Leuchtdiode zum Versenden von Daten und eine Photodiode für den Datenempfang an den vier Seiten des Transportmoduls, wie in Abbildung 14 dargestellt, angebracht. Die oben genannten Dioden sind derart angeordnet, dass sich diese jeweils gegenüberliegen.

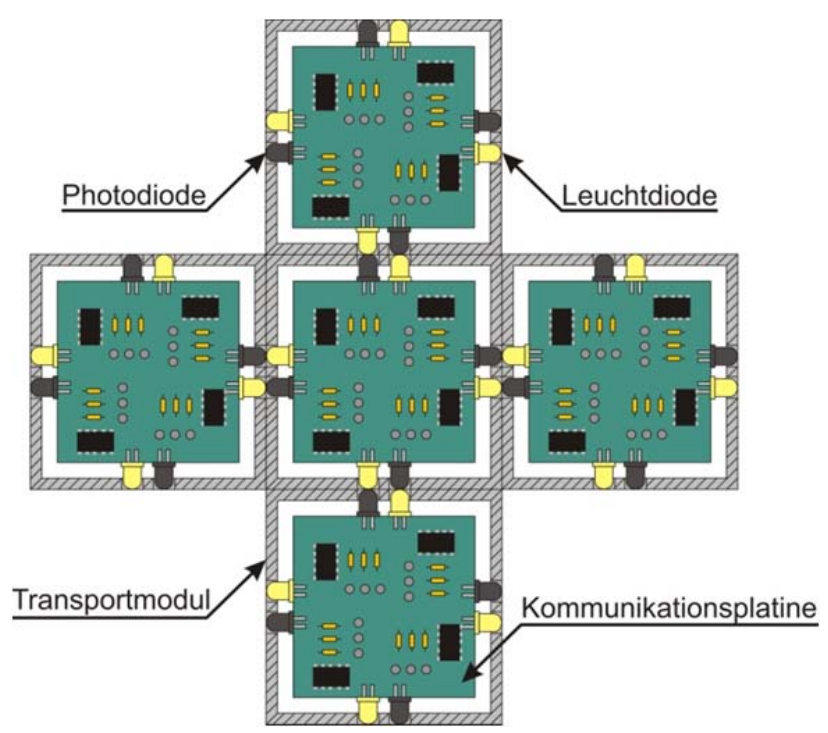

Abbildung 14. Anordnung der Leucht- und Photodioden zum Datenaustausch

Die Steuerungsplatine bekommt durch ein Nachbarmodul über die Kommunikationsschnittstelle ein Signal der eingetroffenen und der zu befördernden Transportein- heit. Nach der Signalverarbeitung im Mikrocontroller wird das Anlaufen des Förderantriebs sowie die Schwenkung in die gewünschte Position veranlasst.

\section{ZUSAMMENSCHLUSS KLEINSKALIGER MODULE ZU HOCHFUNKTIONALEN INTRALOGISTIK-KNOTEN}

Durch die hohe Funktionalität einer Matrix, bestehend aus dem Zusammenschluss omnidirektionaler Module, ist ein einfacher Ansatz bei der Planung intralogistischer Systeme möglich. Die komplexen Funktionen konzentrieren sich in einigen wenigen multifunktionalen Knoten, die mit einfacher Fördertechnik verbunden sind. Die komplexen Sortier- und Verteilaufgaben werden durch diese Knotenpunkte autonom gelöst und die in konventionellen Systemen verteilten Speziallösungen entfallen (siehe Abbildung 15).

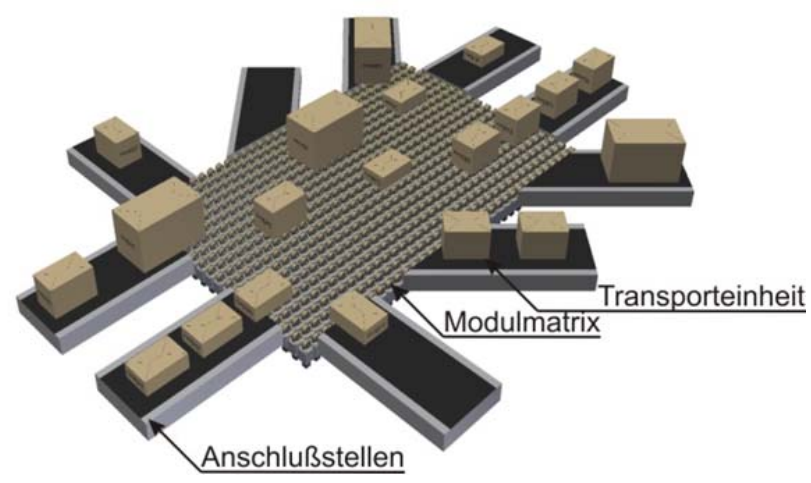

Abbildung 15. Modulmatrix mit Anschlussfördertechnik (beispielhafte Anordnung)

Die Verbindung zwischen den Funktionsknoten kann mit konventioneller, einfacher Fördertechnik geschaffen werden, z. B. Gurtfördertechnik. Abbildung 16 und Abbildung 17 zeigen schematisch die Vernetzung derartiger Funktionsknoten.

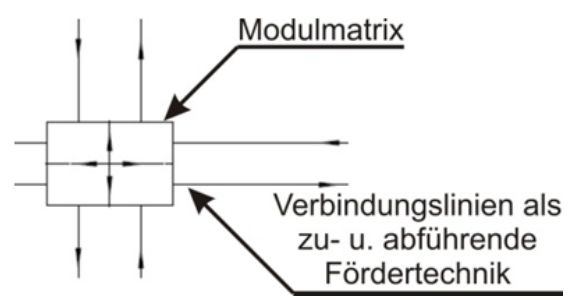

Abbildung 16. Funktionaler Knoten bestehend aus Stetigförderer-Modulen und Anschlussfördertechnik 


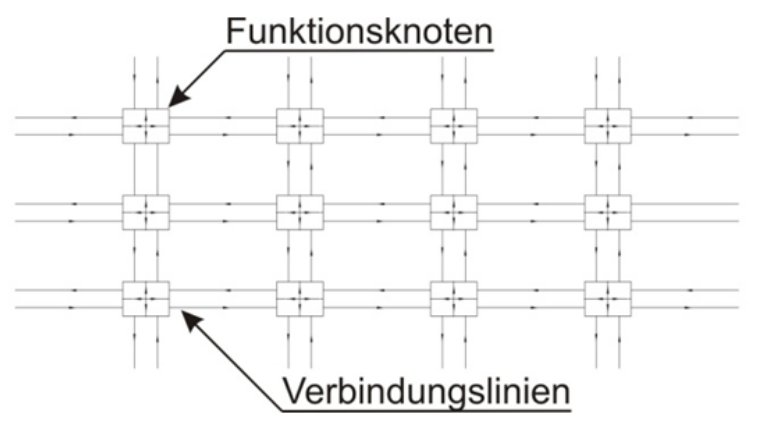

Abbildung 17. Vernetzte funktionale Knoten als übergeordnete Matrix

Diese vernetzte Struktur ist beliebig erweiterbar und in der räumlichen Anordnung äußerst flexibel. Jeder Funktionsknoten lässt sich von jedem anderen Funktionsknoten mit einer Transporteinheit erreichen. Die Vernetzungsstruktur ist darüber hinaus höchst funktional, da sämtliche bekannten Funktionalitäten intralogistischer Systeme in so einer Vernetzung darstellbar sind und zwar genau an dem funktionalen Knoten, wo sie gerade benötigt wird.

\section{ZuSAMMENFASSUNG UND AUSBLICK}

Nachhaltige Trends wie kürzere Produktlebenszyklen und eine Zunahme der Individualisierung fordern wandelbare Lösungen für intralogistische Prozesse [De09].

Die Möglichkeiten der Selbststeuerungsprinzipien in der Intralogistik [De09; Sc09; Fi08] bieten im Zusammenhang mit den Entwicklungsansätzen dezentral gesteuerter Module interessante Lösungsvorschläge, u. a. mit gleichartigen Modulen [FM09] für den Behältertransport.

Dieser Beitrag stellt ein Lösungskonzept auf Basis kleinskaliger Stetigförderer vor. Die kleinskaligen, koppelbaren Module haben eine eigene Antriebstechnik und sind vorbereitet für ein dezentrales Steuerungskonzept mit auf den Modulen verteilten Steuerungsintelligenzen.

Die bisher untersuchten Prototypen sind in Einzelfertigung hergestellt worden, an welchen sich Funktion und Potential des Prinzips zeigen lassen. Für eine Markteinführung ist ein kostensenkendes Serienfertigungskonzept notwendig. Ein wesentlicher Faktor ist hier die Antriebstechnik im Zusammenhang mit der Steuerungselektronik.
Eine mögliche weiterreichende Integration der Antriebstechnik in das kleinskalige Modul ist anhand eines Entwurfs dargestellt. Die integrierte Antriebslösung bietet Möglichkeiten der funktionalen Optimierung und der Kostenreduzierung durch folgende Schritte:

- $\quad$ Antriebs- und Funktionselemente werden vereint

- Antriebselektronik wird für den Bedarf nach Drehmoment und Drehzahl optimiert.

- Ansteureungselektronik und Steuerungsplatinen werden zusammengefasst

- Mechanische Bauteile werden in Serienfertigungsverfahren produziert.

- Elektronische Bauteile werden in Serienfertigungsverfahren produziert.

Idealerweise wird für die Gestaltung der zu integrierenden Antriebstechnik aus Platz- und Kostengründen auf ein Getriebe verzichtet. Dazu ist ein regelbarer Antrieb mit entsprechendem Drehmoment in dem notwendigen Drehzahlbereich von unter 700 1/min notwendig. Passende Konzepte hierfür sind Synchronmotore, ausgeführt als hochpolige Außenläufer. Ein in der Praxis umgesetztes Beispiel in Form eines Transversalflussmotores liefert Cyoris eingesetzt in getriebelosen Rollenantrieben [Cy12].

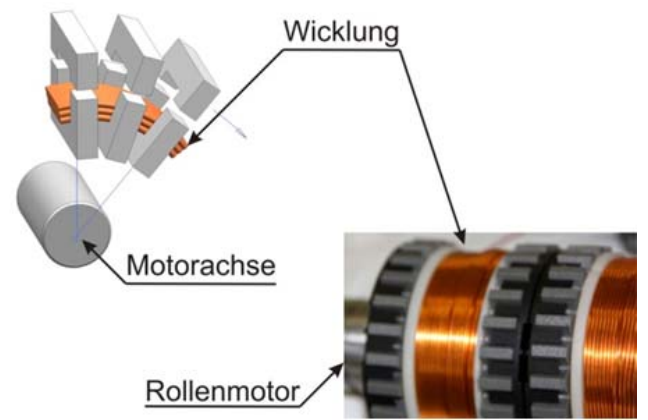

Abbildung 18. Prinzip einen Entwurf der integrierten Antriebe in ein Schwenkrollenmodul

Abbildung 19 zeigt einen Entwurf der integrierten Antriebe in ein Schwenkrollenmodul. Steuerplatinen sind ebenfalls in das Modell integriert, die mechanische Kopplung ist als steckbares Schienensystem ausgeführt und die Datenkommunikation erfolgt opto-elektronisch. Abbildung 20 zeigt schematisch den Zusammenschluß der Module zu einer Funktionsmatrix. Die elektrische Versorgung erfolgt steckbar unterhalb des Moduls. 


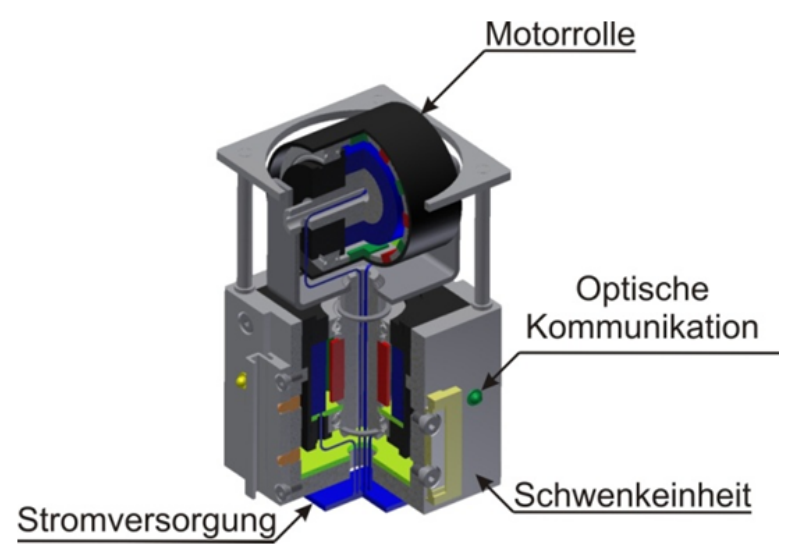

Abbildung 19. Entwurf eines Schwenkrollenmoduls mit integrierter Antriebstechnik in Schnittdarstellung

Die Integration der Antriebstechnik ist analog in das Modul mit dem Schrägscheibenkonzept möglich. Das Prinzip bietet durch nahezu koaxiale Anordnung der Antriebe Möglichkeiten zur kompakteren Bauform und Gehäusegestaltung (siehe Abbildung 20).

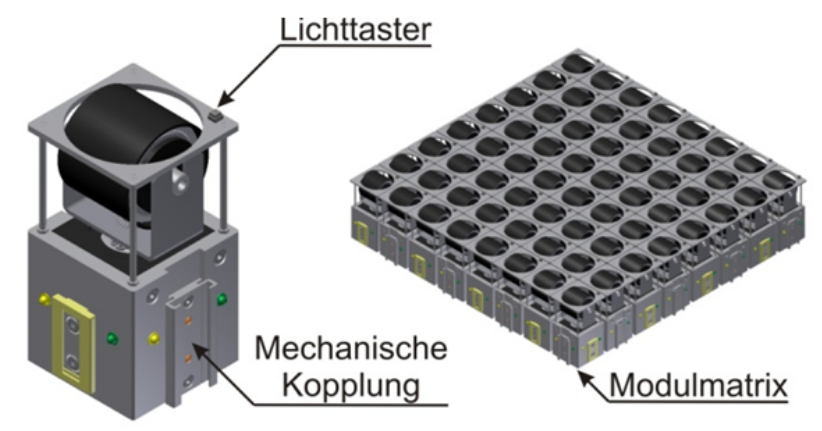

Abbildung 20. Kleinskaliges Modul und Modulmatrix

\section{LITERATUR}

[OKV10] Overmeyer, Ludger; Ventz, Kai; Falkenberg, Sascha; Krühn, Tobias: Kleinskalige multidirektionale Transportmodule für den Einsatz in der Intralogistik; In: 19. Deutscher Materialfluss-Kongress, VDI-Berichte 2094, Düsseldorf: VDI Verlag GmbH, 2010, S.231 - 248, ISBN 978-3-18-092094-8

[Ov07] Overmeyer, Ludger; Falkenberg, Sascha; Heiserich, Gerd; Jungk, Andreas: Innovative Gestaltung von Intralogistik durch Kopplung kleinskaliger Systeme, In: 16. Dt. Materialfluss-Kongress, VDI-Berichte 2007, S. 171-179, ISBN 978-3-18-091978-2

[Ov10] Overmeyer, Ludger; Ventz, Kai; Falkenberg, Sascha; Krühn, Tobias: Interfaced multidirectional small-scaled modules for intralogistics operations, In: Logistics Research 2010, Vol. 2, 123 -133, Springer Verlag 2010, ISSN 1865-035X

[OVF09] Overmeyer, Ludger; Ventz, Kai; Falkenberg, Sascha: Kleinskalige, multidirektionele Transportmodule für den Einsatz in der Intralogistik, In: Logistics Journal, Vol. 1, 2009, ISSN 18607977

[De09] Delfmann, Werner: Entschleunigt, einfach, dezentral - ein Paradigmenwechsel in der Logistik; In: Wimmer, Thomas; Wöhner, Heiko, (Hrsg.): 26. Deutscher Logistik-Kongress, Kongressband, Hamburg: Deutscher Verkehrsverlag, 2009, S. 535 - 554, ISBN 978-3-87154-401-9

[Sc09] Scholz-Reiter, Bernd; Rippel, Daniel, Sowade, Steffen; Teucke, Michael: Selbststeuerung als Ansatz in der Praxis manuell getriebener Logistik. In: Wimmer, Thomas; Wöhner, Heiko, (Hrsg.): 26. Deutscher LogistikKongress, Kongressband, Hamburg: Deutscher Verkehrsverlag, 2009, S. 535 - 554, ISBN 978-3-87154-401-9

[Cy12] CYORIS AG: Datenblätter Cydrive 50, http://www.cyoris.com

[Fi08]

Fischer, Stefan: Naturinspirierte Verfahren in der Informatik - Anregungen für die Logistik. In: Baumgarten, Helmut, (Hrsg.): Das Beste der Logistik Innovationen, Strategien, Umsetzungen, Berlin, Heidelberg: Springer Verlag, 2008, S. 139 - 144, ISBN 978-3-54078404-3

[FM09] Furmans, Kai; Mayer, Stephan: Vollständig dezentraler und autonomer Flexförderer. In: Hebezeuge Fördermittel; Fachzeitschrift für Technische Logistik, Ausgabe 6•2009 